\title{
ОБЗОРЫ
}

\section{Первичные публичные размещения акций: основные исследуемые аномалии ${ }^{31}$}

\author{
Кокорев Д.А. ${ }^{32}$, Чистяков А.Г. ${ }^{33}$
}

В данной статье представлен обзор исследований, посвященных анализу первичных размещений акиий (IPO). Подробно рассматриваются следующче аномалии IРО недооиенка IPO (феномен существенного роста изен на акиии после их размещения), пониженная долгосрочная доходность акций после IPO (на долгосрочном горизонте акиии компании, прошедших через IPO, показывают худшую доходность, чем аналоги) и волны IPO (эмпирически установлена ичикличность размещений). Посредством обзора статей показано, что все три аномалии тесно взаимосвязаны между собой.

\section{$J E L: G 24$}

Ключевые слова: IPO, недооченка IPO, волны IPO, пониженная долгосрочная доходность IPO

\section{Введение}

Наблюдение за рынком США показало, что количество проводимых размещений меняется из года в год: так, в одни годы может быть проведено более 400 IPO, а в другие менее 100. Более того, было замечено систематическое повышение цены акции в первый день торгов: в среднем котировки акций росли на 17,9\% к вечеру первого торгового дня ${ }^{34}$. Это явление в литературе получило название «первичная недооценка акций». Однако в долгосрочной перспективе IPO проигрывают по сравнению с рынком и акциями аналогичных компаний, которые уже котировались на момент проведения IPO (феномен пониженной долгосрочной доходности). Например, за период 1980-2009 трехлетняя доходность IPO в среднем была ниже рыночной на 19,6 процентных пунктов и ниже, чем доходность компаний-аналогов на 7,2 процентных пунктов. Подобные наблюдения дали толчок большому объему теоретических и эмпирических исследований.

Изучение аномалий IPO привлекло внимание многих исследователей по ряду причин. Во-первых, большое количество IPО в периоды роста рынка наводит на мысль, что эмитенты используют так называемые «окна возможностей» (периоды, когда инвесторы излишне оптимистичны и имеет место переоценка компании рынком). Во-вторых, аномалии доходности акций после размещения ставят под сомнение гипотезу об эффективности рынка, согласно которой в цене акции отражается вся доступная информация, а значит, акции аналогичных компаний, с аналогичным риском, должны обладать одинаковой доходностью. Наконец, высокая доходность в первые дни торгов говорит о том, что при размещении IPO эмитент не использовал возможности рынка полностью, установив цену размещения ниже

\footnotetext{
31 Данный обзор был составлен в рамках работы над проектом «Исследования корпоративных финансовых решений компаний России и других стран с развивающимися рынками капитала в условиях глобальной трансформации рынков капитала и становления экономики инновационного типа» Программы фундаментальных исследований НИУ ВШЭ в 2011-2012 годах.

${ }^{32}$ Стажер-исследователь лаборатории корпоративных финансов НИУ ВШЭ.

${ }^{33}$ Бакалавр факультета экономики НИУ ВШЭ.

34 Данные основаны на выборке IPO, проведенных на рынке США за 1980-2011 годы, и взяты с сайта: http://bear.warrington.ufl.edu/RITTER.
} 
оценки стоимости акции рынком.

В данном обзоре мы постараемся систематизировать ключевые исследования упомянутых аномалий IPO.

\section{1. Окна возможностей}

Существование особенностей эмиссионной активности, которые выражаются в большом числе размещений в одни годы и гораздо меньшем - в другие, наталкивает на мысль о существовании «окон возможностей» на рынке капитала. Исследования показали, что на рынке капитала бывают периоды, когда инвесторы подвержены излишнему оптимизму, который, как заметил Риттер (Ritter, 1991), служит стимулом для выхода компаний на IPO. В такие периоды рынок переоценивает компанию, а значит, она может привлечь больший объем средств, чем тот, на который в действительности может претендовать.

Таким образом, в зависимости от настроения инвесторов оценка фирмы рынком в каждый момент времени может оказаться заниженной или завышенной. Эти соображения легли в основу ряда теорий, опирающихся на предпосылку об асимметрии информации и объясняющих выбор компаниями времени для проведения IPO.

Так, Лукас и Макдоналд (Lucas и McDonald, 1990) предложили модель, основанную на теории неблагоприятного отбора и показывающую, что менеджеры решат провести IPO в том случае, если знают, что их компания переоценена рынком. Модель базируется на предпосылках, что менеджеры компании обладают большей информацией о ней, чем рынок, и стремятся максимизировать благосостояние акционеров. Как показывает модель, при соблюдении этих предпосылок менеджмент фирмы будет дожидаться таких настроений инвесторов, которые создадут наиболее благоприятные условия для выхода компании на IPO, то есть позволят получить наибольшую цену за акцию.

Хлой с соавторами (Choe, Masulis \& Nanda 1993) также прибегли к теории неблагоприятного отбора для объяснения цикличности в эмиссионной активности на рынке капитала. Они проанализировали более 5000 размещений американских компаний за период 1971-1991 годов, построив модель на показатели делового цикла. Анализ позволил связать колебания эмиссионной активности со стадией делового цикла. Авторы объяснили это тем, что в периоды экономического подъема фирме доступны более выгодные инвестиционные возможности, а значит, больше фирм захотят привлечь финансирование для их реализации, что приведет к большому числу IPO и, как следствие, обострению проблемы неблагоприятного отбора. Но поскольку фирме открыты выгодные инвестиционные возможности, она охотнее понесет издержки неблагоприятного отбора, связанные с эмиссией акций.

Риттер и Велч (Ritter, Welch, 2002) заметили, что реакция эмиссионной активности на состояние рынков капитала происходит с некоторым запаздыванием, и объяснили это частичной рациональностью предпринимателей, которые оценивают компанию, исходя из своих внутренних представлений о бизнесе и не могут моментально среагировать на изменение его оценки рынком. Эта теория позволяет объяснить временной лаг между улучшением конъюнктуры на финансовом рынке и бумом эмиссионной активности. Предприниматели зачастую прибегают к своим внутренним представлениям о фирме при принятии решений. Это подтверждается и в исследовании Тепловой (Теплова, 2008), согласно которому около 30\% менеджеров на развитых рынках капитала применяют субъективные методы оценки барьерной ставки при принятии инвестиционных решений.

Вышеперечисленные теории позволяют прийти к выводу, что финансовые рынки, включая рынки IPO, подвержены влиянию настроений инвесторов. Менеджеры пользуются их чрезмерным оптимизмом, и поэтому число размещений обычно растет во время таких “окон возможностей”. Этот факт играет важную роль в обосновании факторов, влияющих на аномалии доходности IPO после размещения. 


\section{2. Первичная недоценка IPO}

Риттер и Велч (Ritter, Welch 2002) изучили выборку из 6249 компаний, которые провели IPO в США в период 1980-2001 годов, и пришли к выводу, что в среднем доходность первого дня торгов составила 18,8\%. Для сравнения: дневная доходность компаний-аналогов в среднем составила $0,05 \%$.

Первоначально систематический рост цен акций в течение первого дня впервые был до этого изучен в работах Штолла и Курлей (Stoll, Curley 1970) и Ибботсона (Ibbotson, 1975). Авторы предположили, что в основе моделей, объясняющих такую аномально высокую доходность первого дня, лежит гипотеза о том, что на момент размещения акция была недооценена по сравнению с рынком. Отсюда и пошло название этого феномена - «феномен первичной недооценки».

Наиболее популярными оказались теории, апеллирующие к проблеме асимметрии информации. Интересно, что неполная информация носит двоякий характер: эмитент обладает большей информацией о своей фирме, так как является инсайдером, в то время как инвесторы имеют лучшее представление о спросе рынка на акции эмитента.

Первая группа теорий фокусируется на том, что инвестор лучше осведомлен об истинной стоимости компании, что ставит в центр внимания проблему рынка «лимонов». Идея состоит в том, что инвесторы, взявшие на себя более высокий риск, потребуют недооценку в качестве компенсации за него. В рамках данного подхода эмитенты разделяются по качеству, которое не наблюдается рынком напрямую. При этом фирмы низкого качества могут подавать сигналы о том, что они являются компаниями высокого качества, неся при этом так называемые издержки «имитации». Велч (Welch, 1989) приводит в пример компании нефтегазового сектора, где для надлежащей имитации фирма низкого качества вынуждена строить высокотехнологичный нефтепровод, окупаемость которого для нее намного ниже, чем для компании высокого качества, из-за меньшей эффективности операционной деятельности (например, низкое качество нефти в месторождениях или низкие дебиты скважин). Таким образом, низкокачественные компании создают для рынка видимость эффективной и масштабной операционной деятельности. В этом случае недооценка акций при размещении еще больше увеличивает издержки имитации для фирм низкого качества, лишая их стимула подавать ложные сигналы. Вместе с тем фирмы высокого качества могут позволить себе «оставить деньги на столе» в качестве сигнала инвесторам о том, что они являются компаниями высокого качества.

Даже «теряя» деньги при размещении, фирма высокого качества впоследствии может «отыграть» их за счет ряда выгод, которыми не могут воспользоваться фирмы низкого качества. Во-первых, апеллируя к труду Милгрома и Робертся (Milgrom, Roberts, 1986), можно вспомнить о возможности проведения дополнительных размещений. «Оставив деньги на столе» при первичном размещении, компания дает сигнал о своем качестве и к моменту проведения дополнительной эмиссии может установить более высокую цену, чем и компенсировать потери при проведении IPO. Этого же объяснения придерживается Ибботсон (Ibbotson, 1975): фирмы низкого качества не могут воспользоваться привилегией дополнительного размещения из-за того, что к моменту его проведения рынку откроется информация об их качестве.

Велч (Welch, 1989), проанализировав выборку их 1028 IPO, проведенных американскими компаниями в 1977-1982 годах, нашел эмпирическое подтверждение этой гипотезе. Он заметил, что приблизительно треть компаний из выборки провели дополнительное размещение к 1986 году. При этом в среднем дополнительное размещение привлекало сумму, более чем в 3 раза превышавшую первичное размещение.

Аллен и Фалхабер (Allen, Faulhaber, 1989) приводят аргумент, что первичная недооценка может привести к более благоприятной интерпретации дивидендов рынком, что положительно отразится на стоимости компании. Имитирующая компания, напротив, не 
сможет создать достаточный для выплаты дивидендов денежный поток и воспользоваться этой возможностью для покрытия издержек от недооценки.

Тиник (Tinic, 1988) добавил еще один аргумент, заметив, что первичная недооценка оберегает компанию от судебных исков со стороны инвесторов. Хотя его аргументация не нашла строгого эмпирического подтверждения, она объясняет выбор эмитентом недооценки в качестве инструмента для подачи сигнала о своем качестве.

В то время как эта группа теорий породила множество разногласий, а исследователи так и не пришли к окончательному заключению, она позволяет объяснить добровольную недооценку акций компаниями-эмитентами. Стоит ожидать, что если существует возможность подать рынку сигнал о своем качестве каким-либо другим способов, снизив асимметрию информации, то потребность инвесторов в дополнительном сигнале в виде первичной недооценки акций снизилась бы вместе $\mathrm{c}$ необходимостью компании компенсировать им взятые на себя риски. Как показано в работе Эллула и соавторов (Ellul, Pagano, 2006), в качестве сигнальных показателей могут быть использованы возраст компании и величина ее активов.

Другая группа теорий, апеллирующих к асимметрии информации, сосредоточила свое внимание на том, что инвесторы обладают большей информацией о спросе на акции. Эмитент на момент размещения не знает точно, сколько рынок готов заплатить за его акцию, что приводит к возникновению феномена первичной недооценки. Так, Рок (Rock, 1986) рассмотрел модель, в которой часть инвесторов обладала большей информацией, чем другая. Он заметил, что андеррайтер IPO часто получает заявки на сумму, до пяти раз превышающую объем размещения. В этом случае именно он распределяет доли IPO между инвесторами. В то время как информированный инвестор знает истинную стоимость акции, менее информированные инвесторы вынуждены основывать решение на своем представлении о вероятностях получения переоцененной и недооцененной акций.

Альтернативное объяснение в рамках данного подхода было предложено Велчем (Welch, 1992), который предположил существование «информационных цепей»: инвесторы принимают решение о приобретении акций, основываясь на действиях других инвесторов, с которыми андеррайтер осуществлял переговоры раньше. Таким образом, слишком высокая цена может стать причиной провала IPO, если первый инвестор откажется от размещения. В этом случае первичная недооценка акций вызвана попыткой избежать провала IPO. В поддержку этой теории Амихуд с соавторами (Amihud, Hauser, Kirsh, 2001) заметили, что в основном либо величина предложения размещаемых акций больше, чем величина спроса, либо величина спроса намного превышает объем размещения, и лишь несколько IPO в выборке имели небольшой избыточный спрос.

Наиболее убедительной теорией, связывающей недооценку с незнанием эмитентом спроса рынка на его акции, является теория, предложенная Бенвистом и Вильхемом (Benveniste, Wilhelm, 1990), которые учитывают работу инвестиционных банков андеррайтеров IPO. Инвестиционные банки обычно с помощью road show» - мероприятия, во время которого банки ведут переговоры с потенциальными инвесторами о возможных сделках, пытаются как раз оценить спрос рынка на акции эмитента. Но потенциальные инвесторы понимают, что в случае, если они выкажут готовность купить акции по высокой цене, на них будет установлена высокая цена, а значит, андеррайтер должен предложить чтото взамен за правдивое раскрытие инвестором своей готовности платить. Роль платы за честное раскрытие информации и играет недооценка.

Таким образом, теории, основанные на асимметрии информации, приводят к выводу, что чем более прозрачна компания, тем меньше неопределенность относительно будущей цены ее акций и тем ниже необходимость в недооценке. Действительно, репутация компании, ее признание рынком снижает необходимость ей сигнализировать о своем качестве и снижает диверсификацию в осведомленности инвесторов.

Немного в стороне стоит еще один подход к объяснению недооценки - ее можно связать с неопределенностью относительно ликвидности акции после начала торгов - риском 
ликвидности.

Так, Риттер и Велч (Ritter, Welch, 2002) заметили, что ни премия за фундаментальный риск, ни премия за риск ликвидности не могут объяснить, почему эффект наблюдается только в первый день. Действительно, маловероятно, что неопределенность, связанная с ликвидностью или систематическим риском акции, может быть разрешена за один торговый день.

Связь риска ликвидности с недооценкой акции была подробно изучена Эллулом (Ellul, Pagano, 2006). Предложив модель, объясняющую динамику цен в момент размещения акции и начала ее торгов, они дополнили классическую литературу, предположив, что инвесторы в момент размещения акций не знают точно, насколько ликвидным будет вторичный рынок. Поэтому, чтобы их привлечь, им должна быть предложена такая цена, которая компенсировала бы им взятый на себя риск.

С точки зрения межстрановых различий, помимо различий в деловом обычае, которые могут выражаться в разных промежутках времени между размещением акций и началом торгов, существуют различия в развитии экономик стран, стадиях делового цикла, в особенности их юридических систем и развитости институтов (Hearn, 2013).

Как показали Грох и Лихтенштейн (Groh, Liechtenstein, 2011), высокий уровень коррупции говорит о низкой защищенности инвесторов и низком уровне доверия среди населения. Все это усложняет действие рыночных сил и, возможно, вызывает более высокую степень неприятия риска инвесторами, что отражается в увеличении требуемой премии за него, то есть первичной недооценки.

Наконец, Хопп и Дрехер (Ноpp, Dreher, 2013) проанализировали различие в юридических и институциональных системах стран и пришли к выводу, что IPO в странах с более строгой защитой прав инвесторов характеризуются более высоким уровнем недооценки. Это можно объяснить желанием максимально распылить собственность между инвесторами, чтобы сохранить контроль над компанией. Высокая недооценка позволяет стимулировать спрос на акции и увеличить число собственников. В противном случае инвесторы со значительными пакетами акций будут иметь большой вес при принятии решений и смогут навязывать изменение политики. В то время как эта гипотеза была подтверждена в работе авторов, ее анализ для стран БРИКС заключается в сложности оценки степени защищенности прав инвесторов. Клаппер и Лав (Klapper, Love 2004) применили страновые индексы, характеризующие юридическую систему, но сами же отметили отсутствие их значений для Китая, как причину исключения его из выборки.

\section{3. Пониженная долгосрочная доходность IPO}

Феномен пониженной долгосрочной доходности привлек внимание многих исследователей преимущественно в конце XX века. Гипотеза об эффективности рынка предполагает, что как только IPO размещено и начались торги, его акции ничем не отличаются для рынка от любых других, а значит, их цена должна отражать их внутреннюю стоимость. Многие исследования с привлечением большого объема эмпирических данных показали, что в среднем рыночный портфель, в качестве прокси для которого традиционно выбирается рыночный индекс, либо выборка из компаний-аналогов показывают более высокую доходность, чем акции компании, осуществившей IPO.

Вопрос, почему возникает феномен пониженной доходности, остается неразрешенным до конца до сих пор. Существующие попытки его объяснить апеллируют к наличию на рынке капитала периодов, когда инвесторы излишне оптимистичны. Одна из таких попыток объяснить пониженную долгосрочную доходность IPO была предпринята в работе Миллера (Miller, 1977).

Согласно автору, пониженная доходность IPO проистекает из того простого факта, что при его распределении акции получили наиболее оптимистично настроенные инвесторы, которые предлагали за них наибольшую цену. По прошествии времени рынок 
«охлаждается», а индивидуальная оценка акции наиболее оптимистичного инвестора приближается к средней по рынку, что отражается в понижении котировок. Главная проблема подобной аргументации - она не дает ответа на вопрос, на протяжении какого срока IPO показывают пониженную доходность. Согласно исследованиям Риттера (Ritter, 1991), она наблюдается в течение трех лет после эмиссии.

Риттер заметил, что феномен пониженной доходности, в отличие от недооценки, характерен далеко не для всех IPО. Используя показатели доходности, скорректированной на доходность акций и индексов, выбранных в качестве инструментов для сравнения ${ }^{35}$, он рассмотрел выборку из 1526 IPО обыкновенных акций, проведенных в период 1975-1984 годов на рынке США. Исследование показало, что средняя доходность IPO за три года составила $34,47 \%$. Для сравнения: доходность аналогичных компаний, акции которых уже котировались на момент проведения IPO, составила 61,86\%. Однако IPO, проведенные лишь в течение пяти из десяти лет контрольного периода, показали пониженную по сравнению с компаниями-аналогами доходность. Это подтверждает гипотезу о том, что источником феномена пониженной доходности является наличие эффекта настроений на рынке капитала, периодов излишнего оптимизма инвесторов, когда рынок переоценивает выпуски акций. Таким образом, IPO, размещенные в годы, характеризующиеся более высокой эмиссионной активностью, демонстрируют доходность ниже, чем эталон, выбранный для сравнения, так как информация о последующих денежных потоках компании вынудит рынок скорректировать свою оценку.

Шульц (Schultz, 2001) также прибегает к схемам в эмиссионной активности для объяснения феномена пониженной долгосрочной доходности. Он предложил модель, которая предсказывает результаты, схожие с теми, что описал Риттер (Ritter, 1991). Согласно его модели, компании выходят на рынок, когда их акции оцениваются особенно высоко. При этом сами эмитенты не осознают, что котировки находятся на пиковом уровне. Если же цены продолжают расти, проводится все больше IPO. Разумно ожидать, что в этих условиях избыточная доходность IPO будет отрицательной из-за последующей корректировки цен и возврата котировок к средним значениям.

Из теории Шульца (Schultz, 2001) следует вывод, что если бы доходность IPO абсолютно коррелировала с рыночным индексом, то пик цен на акции приходился бы и на пик рыночного индекса. В случае абсолютной корреляции акции с рыночным индексом феномен пониженной долгосрочной доходности не наблюдался бы, так как избыточная доходность была бы равна нулю. И наоборот, чем сильнее динамика цены акции отличается от динамики рыночного индекса, тем ниже избыточная доходность, так как пик котировок акции, когда происходит бум эмиссионной активности, не совпадает с пиком рыночного индекса, и последующая корректировка приводит к отрицательным избыточным доходностям. В качестве показателя степени рассогласованности котировок акции и рыночного индекса можно использовать волатильность избыточной доходности.

\section{Заключение}

Данная статья систематизирует основные работы по трем ключевым аномалиям IPO: волнам размещений, недоценке и пониженной долгосрочной доходности IPO.

Рассмотренные статьи по цикличности размещений позволяют прийти к выводу, что финансовые рынки, включая рынки IPO, подвержены влиянию настроений инвесторов, и менеджмент компаний старается использовать их чрезмерный оптимизм, что приводит к росту количества размещений во время «окон возможностей».

Согласно теориям асимметрии информации (к которым в основном прибегают исследователи для объяснения аномалий доходности IPO), повышение прозрачности компании снижает неопределенность относительно будущей цены ее акций. тем самым

\footnotetext{
35 Далее: показатели избыточной доходности.
} 
уменьшая и необходимость в недооценке. Так, ключевым фактором снижения асимметрии информации является размер компании (при размещении крупных IPO эмитенты вынуждены раскрывать больше информации из-за более тщательной проверки со стороны потенциальных инвесторов).

Пониженная долгосрочная доходность большинством авторов связывается с тем фактом, что компании стараются выйти на рынок в моменты, когда их акции переоценены, т.е. используя «окна возможностей». Также исследования показывают отрицательную зависимость между уровнем недооценки и долгосрочной доходностью акций. Таким образом, можно заключить, что все три аномалии IPO тесно взаимосвязаны между собой.

\section{Список литературы}

1. Теплова Т.В. Тестирование практики построения прогнозного бета-коэффициента в конструкции САРМ с учетом низкой ликвидности ценных бумаг на российском рынке // Аудит и финансовый анализ. 2004. № 4.

2. Теплова Т.В. Инвестиционные рычаги максимизации стоимости компании. Практика российских предприятий. М: Вершина, 2008.

3. Allen, F., and Faulhaber, G.R. (1989), Signaling by Underpricing in the IPO Market, Journal of Financial Economics, 23 (1989) 303-324.

4. Amihud, Y, Shmuel. H., and Kirsh, A. (2001), Allocations, Adverse Selection and Cascades in IPOs: Evidence from Israel, Working paper, New York University.

5. Benveniste, L. M., and Wilhelm, W.J. (1990), A Comparative Analysis of IPO Proceeds Under Alternative Regulatory Environments, Journal of Financial Economics, 28 (1990) 173-208.

6. Booth, J.R., and Chua, L. (1996), Ownership Dispersion, Costly Information, and IPO Underpricing, Journal of Financial Economics, 41 (1996) 291-310.

7. Choe, H., Masulis, R., and Nanda, V. (1993), Common stock offerings across the business cycle: Theory and evidence, Journal of Empirical Finance, 1 (1993) 3-31.

8. Groh, L, (2011), International allocation determinants for institutional investments in venture capital and private equity limited partnerships, International Journal of Banking, Accounting and Finance, 2-3(3) (2011).

9. Hearn B. (2013), The institutional determinants of IPO firm prospectus length in a developing context: A research note, Research in International Business and Finance, 1(27) (2013).

10. Hickman, W. (1953), The Volume of Corporate Bond Financing Since 1900, National Bureau of Economic Research, Inc.

11. Hopp, C. Dreher, A. (2013), Do differences in insrirurional and legal environments explain cross-country variations in IPO underpricing?, Applied Economics, 45:4 (2013), 435-454.

12. Klapper, L., Love I. (2004), Corporate Governance, Investor Protection, and Performance in Emerging Markets, Journal of Corporate Finance, 10 (2004), 703-728.

13. Lucas, D., McDonald R. (1990), Equity Issues and Stock Price Dynamics, The Journal of Finance, 45 (1990) 1019-1043.

14. Ellul A., Pagano M. (2006), IPO Underpricing and After-Market Liquidity, The Review of Financial Studies, 19(2006) 381-421.

15. Ibbotson, R. (1975), Price Performance of Common Stock New Issues, Journal of Financial Economics, 2 (1975) 235-272.

16. Narasimhan, J., Weinstein, M., Welch, I. (1993), An Empirical Investigation of IPO Returns and Subsequent Equity Offerings, Journal of Financial Economics, 34(1993) 153-175.

17. Milgrom, P., Roberts J. (1986), Price and Advertising Signals of Product Quality, Journal of Political Economy, 94 (1986) 796-821.

18. Miller, E. (1977), Risk, uncertainty, and divergence of opinion, Journal of Finance, 32 (1977) 1151-1168. 
19. Pagano, M., Panetta F., Zingales L. (1998). Why Do Companies Go Public? An Empirical Analysis, The Journal of Finance, 53 (1998) 27-64.

20. Ritter, J. (1984), The "Hot Issue" Market of 1980, The Journal of Business, 57 (1984) 215240.

21. Ritter, J. (1991), The Long-Run Performance of Initial Public Offerings, The Journal of Finance, 46 (1991) 3-27.

22. Ritter, Ja., Welch I. (2002): A Review of IPO Activity, Pricing, and Allocations, The Journal of Finance, 57, 1795-1828.

23. Rock, K. (1986), Why New Issues Are Underpriced, Journal of Financial Economics, 15 (1986) 187-212.

24. Schultz, P. (2001). Pseudo Market Timing and the Long-Run Underperformance of IPOs, Working paper, University of Notre Dame.

25. Shiller, R. (1990), Speculative Prices and Popular Models, Journal of Business, 32 (1990) 215-240.

26. Tinic, S. (1988), Anatomy of Initial Public Offerings of Common Stock, Journal of Finance, 43 (1988) 789-822.

27. Welch, I. (1989), Seasoned offerings, imitation costs, and the underpricing of initial public offerings, Journal of Finance, 44 (1989) 421-450.

28. Welch, I. (1992), Sequential sales, learning, and cascades, Journal of Finance, 47 (1992) $695-732$. 